\title{
Analysis And Performance Of A Picosecond Dye Laser Amplifier Chain
}

Koch, T., Chiu, L., Yariv, A.

T. L. Koch, L. C. Chiu, A. Yariv, "Analysis And Performance Of A Picosecond Dye Laser Amplifier Chain," Proc. SPIE 0322, Picosecond Lasers and Applications, (23 April 1982); doi: 10.1117/12.933204

Event: 1982 Los Angeles Technical Symposium, 1982, Los Angeles, United States 


\title{
Analysis and performance of a picosecond dye laser amplifier chain
}

\author{
T. L. Koch, L. C. Chiu, A. Yariv \\ California Institute of Technology, Pasadena, California 91125
}

\begin{abstract}
Design considerations are discussed for a simple, easy to use and relatively efficient high gain dye laser amplifier chain for CW mode-locked dye lasers. The amplifier boosts the output of a synchronously mode-locked dye laser to obtain $\sim 0.5 \mathrm{mj}$, $\leq 1$ psec pulses over a $~$ $400 \AA$ bandwidth. These pulses are suitable for efficient Raman Shiftiñg, frequency mixing and continuum generation to vastly extend the spectral range of the system. our amplifier is pumped by a frequency doubled Nd:YAG oscillator only, which longitudinally pumps three identical brewster cells with the same flowing dye solution in each. Contrary to popular belief, high small signal gains $\left(>10^{5}\right)$ are easily attained in a single stage with longitudinal pumping, with better beam homogeneity and easier alignment than transverse pumping. Gain saturation measurements are presented which agree well with calculations. Factors which relax the pump timing sensitivity are examined. The importance of gain saturation for both efficient amplification and for amplitude stability is also discussed. The need for isolated amplifier stages is stressed and optimal amplifier cell areas for a given stage are calculated.
\end{abstract}

\section{Introduction}

Over the past several years, a number of investigators have developed picosecond laser systems which incorporate dye laser amplifiers to boost the energy of CW mode-locked dye lasers ${ }^{1-5}$. These systems successfully couple the many advantages of ch mode-locked dye lasers with the higher energies available from solid state lasers to create a very versaile experimental apparatus. However, relatively few publications, ${ }^{6}$ have appeared which specifically address design problems peculiar to picosecond amplifier systems. Below, we first describe our laser amplifier set up and briefly discuss its characteristics. A relatively simple mathematical model, to be developed more fully elsewhere ${ }^{8}$, is presented which permits a discussion of a number of processes which strongly influence the design of a picosecond amplifier system for CW mode-locked dye lasers.

\section{Experimental Apparatus}

It is well confirmed that the short energetic pulses from a frequency doubled, $0-$ switched Nd:YAG laser provice an almost ideal pump for dye amplifiers in the $6000 \AA$ region of the spectrum. While most systems reported employ ${ }^{-4}$ costly Nd:YAG oscillator-amplifier combinations with multiple doubling crystals, we find that a single oscillator and doubling crystal is sufficient to insure outputs on the order of $0.5 \mathrm{millijoule.} \mathrm{The} \mathrm{Nd:YAG} \mathrm{model} \mathrm{we}$ employed, chosen for its excellent temporal triggering stability and good spatial beam profile, provided $-75 \mathrm{mj}$ at $532 \mathrm{~nm}$ in a $-15 \mathrm{nsec}$ pulse. While there was a pronounced modebeating structure on the pulse, it was very reproducible from pulse to pulse and only a relatively short portion of the pulse is effectively used anyway.

Our rhodamine 8G dye oscillator was pumped by a mode-locked argon ion laser and used both a brewster prism and a wedge interference filter for tuning and limiting the bandwidth. The output pulsewidth was monitored with a real time rotating prism autocorrelation device, while the spectrum was monitored with an optical multichannel analyzer. The RF driver for the argon mode-locker was counted down with appropriate delays to drive the flashlamp and Q-switch of the Nd:YAG laser with subnanosecond timing precision.

The amplifier system is shown in Fig. 1. The $\sim 8 \mathrm{~mm}$ diameter 1.06 um output beam is rotated by a half wave plate HWP to $45^{\circ}$ polarization and then telescoped by Tl to $5.5 \mathrm{~mm}$ diameter to insure good doubling efficiency (>35\%) in the $5 \mathrm{~cm}$ type II KDP crystal which follows and attain the diameter appropriate for the third amplifier stage. Harmonic beamsplitters $\mathrm{HBT}-3$ remove $\sim 99.8 \%$ of the fundamental and beamsplitter BS1 sends $80 \%$ of the energy down a delay line to the third stage A3, while the remaining $20 \%$ is telescoped by 12 to $-2.5 \mathrm{~mm}$ diameter and split approximately in half by BS2 and sent to the first and second stages Al and A2, again with appropriate delays. As can be seen, all three stages are longitudinally pumped. We believe this provides nigher gains, better beam homogeneity and easier alignment than transverse pumping. The $532 \mathrm{~nm}$ pump is reflected into and out of the beam path using dichroic mirrors D1-6 which pass most of the signal. The three flowing dye cells are identical $1.8 \mathrm{~cm}$ bore clear tubes with nickel-plated brass end caps to hold the fused quartz brewster windows. This permits the use of high quality windows and also allows easy and economical replacement of damaged windows. The system design allows the 


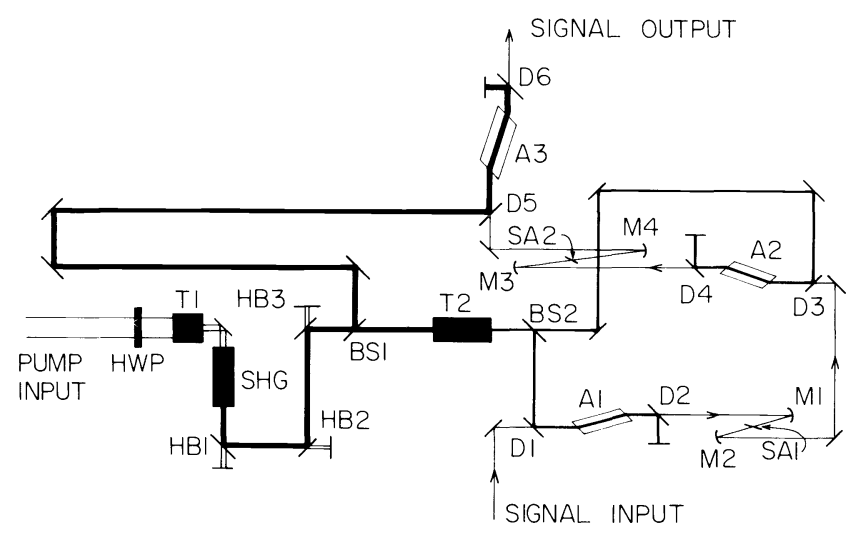

Figure 1. Experimental configuration of amplifier system

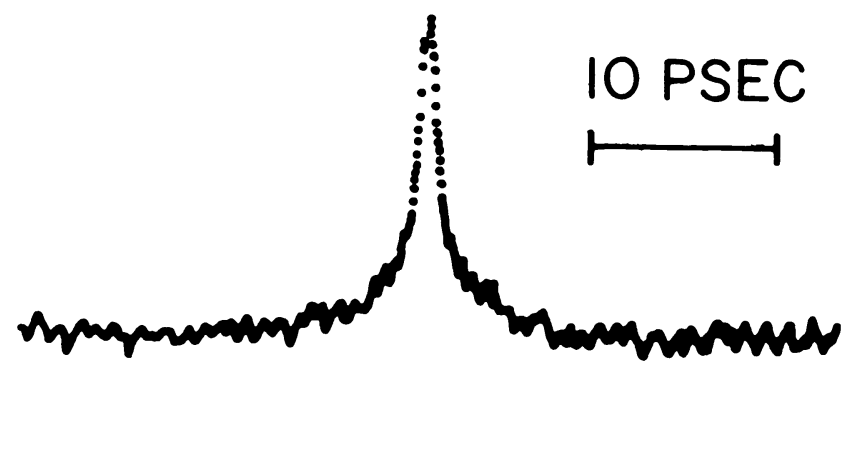

Figure 2. Typical autocorrelation of amplifier output with a FWHM of 1.36 psec or a sech ${ }^{2}$ pulse width of 0.88 psec

same ( 1 10-5 M Rhodamine 640 in methanol) solution of dye to flow in all three cells with one low capacity gear pump. Saturable absorbers SAl-2 are identical flowing ethylene glycol jets with a high concentration of malachite green having a small signal transmission of $\leq 10^{-7}$ at $630 \mathrm{~nm}$. Mirrors $M 1$ and $M 2$ are $5 \mathrm{~cm}$ radius of. curvature while M3 and M4 are $20 \mathrm{~cm}$ and $50 \mathrm{~cm}$ respectively to expand the beam to the appropriate diameter for the last stage. Reflecting optics and brewster angles at all interfaces are used to eliminate any reflections which would limit the gain in a given stage.

The output of the amplifier system has energy up to $0.7 \mathrm{mj}$ with no pulse broadening unless the saturable absorbers are intentionally misadjusted by a large amount. The large small signal attenuation of SAl-2 provides good control of the leading edge to prevent pulse broadening ${ }^{9}$ but still passes $~ 75 \%$ of the pulse energy. Fig. 2 shows a typical autocorrelation of the output pulse, having a FWHM of 1.36 psec or a pulsewidth of 0.88 psec if we assume a sech ${ }^{2}$ pulse shape.

\section{Mathematical Model}

To analyze the gross behavior of a given amplifier cell, we employ as a first approximation of a four-level dye model with infinitely fast relaxation to the upper lasing level $\mathrm{N}_{2}$, and from the lower lasing level to the ground state $\mathrm{N}_{1}$ where $\mathrm{N}_{1}+\mathrm{N}_{2}=\mathrm{N}_{\text {, the total dye }}$ concentration. Neglecting group velocity dispersion and assuming the transverse relaxation time $T_{2}$ is much shorter than times of interest, we write the incoherent traveling wave rate equations:

$$
\begin{aligned}
& \frac{\partial \phi_{1}}{\partial t}+v_{g} \frac{\partial \phi_{1}}{\partial x}=v_{g}\left(\sigma_{1}^{e m_{1}} N_{2}-\sigma_{1}^{a b s_{N_{1}}}\right) \phi_{1} \\
& \frac{\partial \phi_{2}}{\partial t}+v_{g} \frac{\partial \phi_{2}}{\partial x}=v_{g}\left(\sigma_{2}^{e m_{N}} N_{2}-\sigma_{2}^{a b s_{N}}\right) \phi_{2} \\
& \frac{\partial N_{1}}{\partial t}=v_{g}\left(\sigma_{1}^{e m_{\phi_{1}}}+\sigma_{2}^{e m_{\phi_{2}}}\right) N_{2}-v_{g}\left(\sigma_{1}^{a b s_{\phi_{1}}}+\sigma_{2}^{a b s_{\phi_{2}}}\right) N_{1}+\frac{N_{2}}{\tau} \\
& \frac{\partial N_{2}}{\partial t}=-\frac{\partial N_{1}}{\partial t}
\end{aligned}
$$

Here $\phi_{1,2}$ are the pump and signal photon densities, $v_{g}$ is the group velocity in the solvent, and $\sigma_{1}^{e m}, 2$ and $\sigma_{1}^{a b s}$ are the emission and absorption cross-sections at the pump (1) and 
signal (2) frequencies, and $\tau$ is the relaxation time of the dye.

Although non-linear, these equations are readily combined and integrated in space over the cell length $L$ to yield the ordinary differential equation

$$
\begin{aligned}
\frac{d G}{d t}=v_{g}\left(\sigma_{2}^{e m}\right. & \left.+\sigma_{2}^{a b s}\right) G\left[\phi_{10}(t)\left(1-e^{-k N L} G^{\gamma}\right)-\phi_{20}(t)(G-1)\right] \\
& -\frac{1}{\tau} G\left[\ln G+\sigma_{2}^{a b s} N L\right]
\end{aligned}
$$

where $G$ is the appropriately retarded gain

$$
G(t)=\frac{\phi_{2}\left(t+\frac{L}{v_{g}}, L\right)}{\phi_{20}(t)}
$$

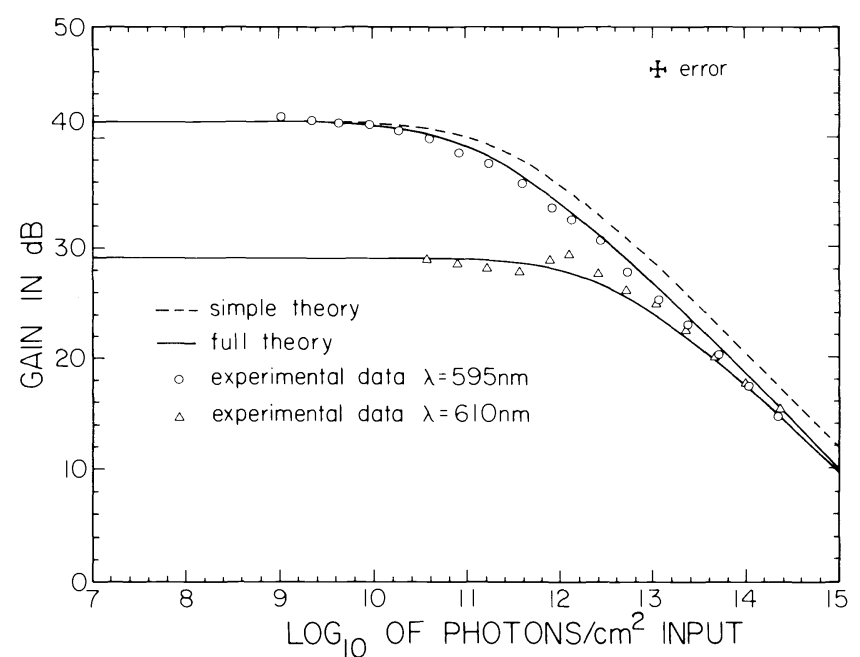

Figure 3. Third stage saturation behavior at $595 \mathrm{~nm}$ and $610 \mathrm{~nm}$. Dashed curve represents EQ. ( 3 ); for solid curve see text. Linear losses are included in measured values, so actual gain is higher.

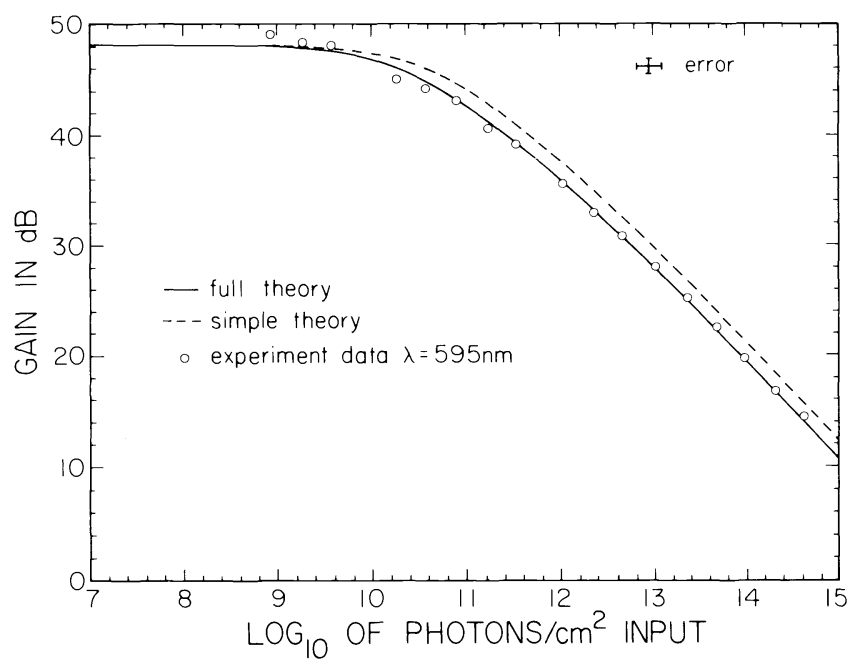

Figure 4. Second stage saturation behavior at $595 \mathrm{~nm}$. Dashed curve represents Eq. (3); for solid curve see text. Linear losses are included in measured values, so actual gain is higher.

and $\phi_{10}(t)$ and $\phi_{20}(t)$ are the pump and signal photon densities at the entrance plane. Here

$$
\kappa=\frac{\left(\sigma_{2}^{\mathrm{em}} \sigma_{1}^{\mathrm{abs}}-\sigma_{2}^{\mathrm{abs}} \sigma_{1}^{\mathrm{em}}\right)}{\left(\sigma_{2}^{\mathrm{em}}+\sigma_{2}^{\mathrm{abs}}\right)}
$$

and

$$
\gamma=\frac{\left(\sigma_{1}^{e m}+\sigma_{1}^{a b s}\right)}{\left(\sigma_{2}^{e m}+\sigma_{2}^{a b s}\right)}
$$

For convenience of discussion, we split Eq. (2) into the pumping and extraction processes, which is equivalent to the statement that negligible pumping occurs during the signal pulse duration. Thus for the amplification process itself, we 1 et $\tau \rightarrow \infty$, $\phi 10(t) \rightarrow 0$ and obtain the well known solution ${ }^{10}$

$$
G(t)=\left[1+e^{-F(t)}\left(\frac{1}{G_{0}}-1\right)\right]^{-1}
$$


where

$$
F(t)=v_{g}\left(\sigma_{2}^{e m}+\sigma_{2}^{a b s}\right) \int_{-\infty}^{t} \phi_{20}(t) d t
$$

which yields the well known result for the net gain

$$
G_{\text {actual }}=\frac{1}{S} \ln \left(1+G_{0}\left(e^{S}-1\right)\right)
$$

Here

$$
S=F(t=\infty)=\frac{E_{s i g}\left(\sigma_{2}^{e m}+\sigma_{2}^{a b s}\right)}{A h \nu \operatorname{sig}}
$$
where Esig the signal energy and $A$ is the signal beam area. Go as used above is now an
"initiajigondition" gain given by integrating for Go $(t)$ using

$$
\frac{d G_{0}}{d t}=v_{g}\left(\sigma_{2}^{e m}+\sigma_{2}^{a b s}\right) G_{0} \phi_{10}(t)\left(1-e^{-k N L} G_{0}^{\gamma}\right)-\frac{1}{\tau} G_{0}\left(\ln G_{0}+\sigma_{2}^{a b s} N L\right)
$$

and evaluating $G_{0}(t)$ at the arrival time of the signal pulse.

\section{Gain Saturation}

Fig. 3 and Fig. 4 show experimental results for gain saturation. Fig. 3 is from the third stage of our system and the 0 and $\Delta$ represent $595 \mathrm{~nm}$ and $610 \mathrm{~nm}$ gains, respectively, as a function of input pulse energy. Fig. 4 shows the $595 \mathrm{~nm}$ gain for the second stage of our system. These measured gains include linear losses from the dichroic mirrors before and after the amplifier cell, so the actual gain is higher: For the theoretical curves, the small signal gains were fit to the experimental small signal gain, but the saturation behavior was dictated by the rate equation analysis. Cross sections were reduced from the data of Batchelder ${ }^{1}$. The dashed 1 ines for the $595 \mathrm{~nm}$ results represent Eq. ( 3 ) and as might be expected, there is only qualitative agreement. The solid lines represent a more sophisticated analysis which includes angular hole burning and triplet losses ${ }^{8}$. The agreement with the latter is very good.

Gain saturation is important for efficient amplification, and it is also desirable for amplitude stability. Since the small signal gain (neglecting saturable absorbers) for our system is - $140 \mathrm{~dB}$, the gain is severely saturated. A small decrease in the input signal, for example, will not affect the output much since in the first two stages the signal still achieves the saturation intensity necessary to extract the majority of the stored inversion energy in the last stage. The saturable absorbers tend to reduce this effect, but we have still observed a significant decrease in amplitude variation upon traversal through the system; $50 \%$ reduction in input typically causes only a 10\% or less reduction in the output. This behavior also makes the actual gain much more flat across the spectrum than an exponential dependence of small signal gain on cross section would suggest.

\section{Pumping and Amplified Spontaneous Emission}

To analyze the pumping process, we note that Eq. (4) leaves out the depleting effect of amplified spontaneous emission (ASE). This can be included to a good approximation by using Eq. (4) for the gain at the peak wavelength, but letting $\tau \rightarrow$ Teff $^{\text {where }}{ }^{8}$

$$
\frac{1}{\tau_{\text {eff }}}=\frac{1}{\tau}\left[1+\frac{1}{8} \frac{D^{2}}{L^{2}} G_{0}\left(\ln G_{0}\right)^{-\frac{3}{2}}\left(1+\frac{4}{\ell n G_{0}}+\frac{18}{\left(\ell n G_{0}\right)_{2}}+. . .\right)\right]
$$

which is an asymptotic result assuming $\ell$ ngo $>1$ and $L \gg D$ where $D$ is the cell active diameter. Teff includes the depletion from induced emissions caused by the ASE flux. With this modification, Eq. (4) gives the gross features of the longitudinal pumping process. To reduce the timing sensitivity, it is desirable to achieve a steady state, which inherently requires energy dissipation after a certain point in time. There are two important features which accomplish this and effectively put a "ceiling" on the gain. One is the strong dependence of teff on gain and the second is the fact that once the pump has longitudinally bleached al the way through the dye cell, no further gain can result. Both of these effects make a "steady state" obtain rapidly and make pulse timing less critical. Fig. 5 shows the peak gain of the first stage of our system from a numerical integration of 
an equation similar to Eq. (4) with $\tau \rightarrow$ Teff but including some allowance for angular hole burning and triplet losses ${ }^{8}$. The gain almost follows the pump linearly and experimental monitoring of the on-axis ASE power, which is proportional to $\left.G_{0}\left(\ln G_{0}\right)\right)^{-\frac{1}{2}}$ confirms this ${ }^{8}$.

\section{Efficiency Optimization}

To analyze the efficiency of a cell, we consider steady state solutions since we are only interested in trends. The factor (1 - e-kNLGr) in Eq. (4) represents the fraction of pump power absorbed and we assume that as we vary all quantities below, we always adjust the dye concentration $N$ so that a good fraction of the pump power is absorbed, i.e., $\left(1-e^{-K N L} G_{0}^{Y}\right)=$ const. $=f$. We then obtain

$$
G_{0}=C_{1} \exp \left[\frac{C_{2}}{A\left(1+\frac{A G_{0}}{2 \pi L}\left(\ln G_{0}\right)-\frac{3}{2}\left(1+\frac{4}{\ln G_{0}}+\frac{18}{\left(\ln G_{0}\right)^{2}}+. . .\right)\right)}-C_{3} \ln G_{0}\right]
$$

and we have from Eq. (3)

$$
G_{\text {actua } 1}=\frac{A}{C_{4}} \ln \left(1+G_{0}\left(e^{C_{4} / A}-1\right)\right)
$$

where $C_{1}, C_{3}$ are constants depending only on $f$ and cross sections, and $C_{2}$ and $C_{4}$ are conare constants proportional to pump power and signal energies, respectively, and $A$ is the cross-sectional area of the active region of the cell. We assume here a perfect match between signal and pump beam diameters.

Fig. 6 shows the small signal gain in dB and the actual gain on a linear scale obtained from Eq. (6) and (7) as a function of active diameter for various values of pump energy, all with a fixed signal energy of $1.5 \times 10^{-6}$ joules and a $10 \mathrm{~cm}$ cell. These are typical values for the second stage of our system. While the small signal gain always increases as $D \rightarrow 0$, for the actual gain there is always an optimal diameter for each pump and signal 1evel. This results from a competition between energy storage and energy extraction and

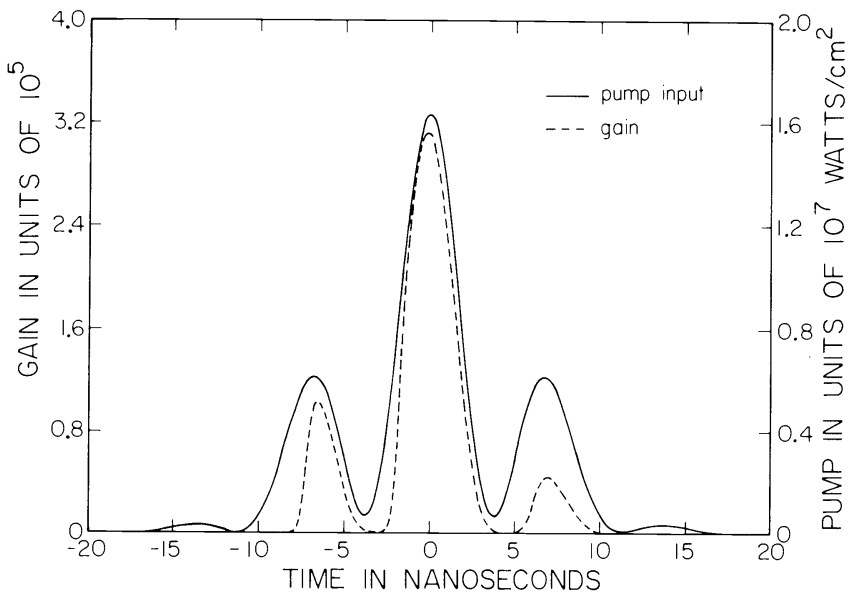

Fig. 5. Transient gain calculation at $595 \mathrm{~nm}$ for first cell including ASE depletion and triplet losses. Pump is measured doubled Nd:YAG power going into first stage.

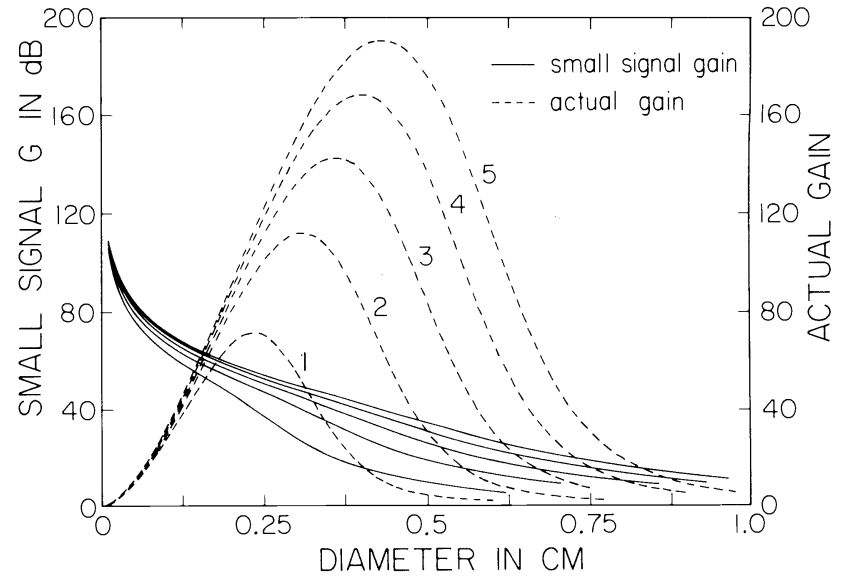

Fig. 6. Calculated small signal and actual gain at $595 \mathrm{~nm}$ as a function of active diameter for input energy of $1.5 \times 10^{-6}$ joule and cell length of $10 \mathrm{~cm}$. Curves 1-5 represent results for pump pulse energies of $2,4,6,8,10 \mathrm{mj}$ respectively.

can be explained qualitatively as follows. The most efficient extraction of a fixed amount of energy occurs when the diameter is as small as possible; the sianal reaches the 
saturation intensity very quickly and extracts almost all of the stored energy. However, to distribute the same total energy $E_{\text {sto }} \cong N_{2} A L h v_{s i g}$ in such a thin cell requires that the small signal gain, which is roughly proportional to $e^{N_{2} \sigma e m L}$ increase as

$$
G_{0} \sim \exp \left(\frac{E_{s t o} \sigma^{e m}}{h \nu_{s i g^{A}}}\right)
$$

as A becomes smaller. For any finite length, Eq. (6) will not let G increase this rapidly because of ASE. As soon as $G_{0}\left(\ell n G_{0}\right)^{-3 / 2} \sim\left(2 \pi L^{2}\right) / A$, further reducti8n in $A$ does not increase $G_{0}$ as quickly as exp( $\left.\left.E_{s t o} \sigma e m\right) /\left(h v_{s i g} A\right)\right)$ any more and the stored energy begins to suffer. The best design, therefore, represents a balance between the best diameter for energy storage and the small diameters needed for efficient energy extraction, and the most efficient cell will be one where ASE is just beginning to play a large role. We can also infer from this argument that the existence of an optimal diameter actually results from practical considerations on cell length, but the length dependence is weak ( $10 \mathrm{garithmic}$ ) so a substantial improvement would require absurdly long cells. In passing, we note an advantage of longitudinal pumping over transverse pumping. Any mismatch between signal beam and active region cross-sectional areas both wastes energy and reduces gain from ASE considerations, since the aspect ratio D/L increases. The spatial characteristics of longitudinal pumping permit a good match without the adverse effects of reflecting and aberrating wall nearby.

To obtain the most extracted energy, we set the cell diameter at the optimal value as we vary all quantities. In principle, we solve Eq. (6) for $G_{0}(A)$, insert this in Eq. (7) and maximize Gactual with respect to A for each pump energy and then see how this varies with pump energy for various values of input signal energy. This is shown in Fig. 7 , all for $L=10 \mathrm{~cm}$ and signal energies ranging from $10^{-9}$ to $10^{-4}$ joule. It is immediately obvious why a multiple cell arrangement with ASE isolation is needed if reasonable efficiency is to be expected. The majority of the energy can then be devoted to the last stage where the storage and extraction problems have a more harmonious solution. We can also conclude that if more pump energy was made available, a fourth stage would be desirable providing linear losses are not too high, but almost comparable results should be obtained if the third stage were increased in area slightly and the energy were deposited there. In any event, only a few mj would be available even for $100 \mathrm{mj}$ of pump energy in a 15 nsec pulse.

\section{Conclusion}

In closing, we have shown that a picosecond dye laser amplifier chain can be constructed using a Nd:YAG oscillator alone with longitudinal pumping of all cells and energies on the order of $0.5 \mathrm{mj}$ are easily attained with excellent beam quality. We have shown that the extreme saturation behavior can be well understood and, when properly employed, that it causes efficient energy extraction, good amplitude stability and negligible pulse broadening. Finally, we have shown how the energy storage problem and the energy extraction problem can be balanced out to obtain the optimal cell geometry for a given stage.

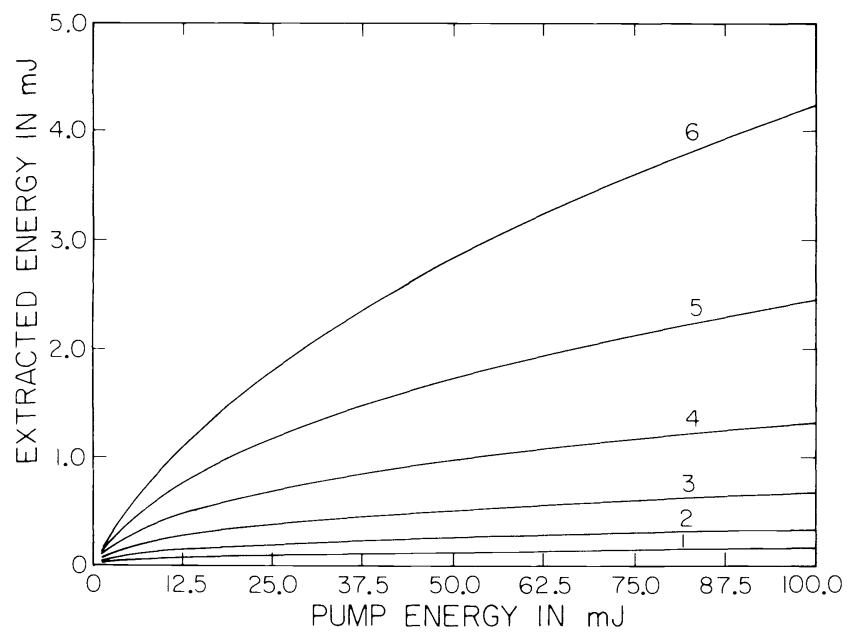

Figure 7

Optimized extracted energy vs. pump pulse energy at $595 \mathrm{~nm}$ using approximated steady state analysis. Curves 1-6 are for signal energies of $10^{-9}, 10^{-8}, 10^{-7}, 10^{-6}, 10^{25}$, and $10^{-4}$ joules respectively'. 


\section{Acknowledgements}

The authors would like to express their gratitude to Desmond Armstrong for his technical assistance in the synchronizing electronics and to Larry Begay for assistance in the construction of the experimental apparatus. We are also indebted to Ti Rong Chen for his assistance in taking experimental data. This work was supported by an N.S.F. grant and by a N.S.F. Graduate Fellowship (T.L.K.).

\section{References}

1. Ippen, E. P. and Shank, C. V., Picosecond Phenomena, Springer Series in Chemical Physics, Vol. 4, Ed. by C. V. Shank, E. P. Ippen and S. L. Shapiro, Springer-Verlag, Berlin, Heidelberg, New York, pp. 103. 1978.

2. Martin, J. L., Astier, R., Antonetti, A., Minard, C. A., and Orszag, A., C. R. Acad. Sc. Paris, t. 289, 45. 1979.

3. Rotman, S. R., Roxio, C. B., Bebelaar, D., Yee, T. K., and Salour, M. M., Picosecond Phenomena II, Springer Series in Chemical Physics, Vol. 14, Ed. by R. M. Hochstrasser, W. Kaiser and C. V. Shank, Springer-Verlag, Berlin, Heidelburg, New York, pp. 50.

4. Matsuoka, M., Nakatsuka, H., and Fujita, M., Picosecond Phenomena II, Springer Series in Chemical Physics, Vol. 14, Ed. by R. M. Hochstrasser, W. Kaiser and C. V. Shank, Springer-Verlag, Berlin, Heidelberg, New York, pp. 357. 1980.

5. Kryukov, P. G., llatveets, Yu. A., and Semchishen, V. A., Sov. J. Quantum Electron., Vol. 10, pp. 1418. 1980 .

6. Ippen, E. P., Shank, C. V., Wiesenfeld, J. M., Migus, A., Phil. Trans. R. Soc. Lond., Vo1. A298, pp 225. 1980

7. Migus, A., Martin, J. L., Astier, R., and Orszag, A., Picosecond Phenomena II, Springer Series in Chemical Physics, Vol. 14, Ed. by R. M. Hochstrasser, W. Kaiser and C. V. Shank, Springer-Verlag, Berlin, Heidelburg, New York, pp. 59. 1980.

8. Koch, T. L., Chiu, L. C. and Yariv, A., to be submitted for publication.

9. Basov, N. G., and Letokhov, V. S., Sov. Phys. Doklady, Vol. 11, pp. 222.1966.

10. Frantz, L. M., and Nodvik, J. S., J. Appl. Phys., Vol. 34, pp. 2346.

11. Private communication with S. Batchelder, May, 1981. 